\title{
Entre la teoría y la práctica de la conservación de los recursos naturales: las parcialidades' de Totonicapán, Guatemala
}

\author{
Between theory and practice of conservation of natural \\ resources: the parcialidades of Totonicapán, Guatemala
}

\author{
Luis Arturo Sánchez-Midence ${ }^{2}$ \\ Tomás de Jesús Guzmán ${ }^{3}$
}

Fecha de recepción: 22 de noviembre del 2012

Fecha de aprobación: 22 de febrero del 2013

Sánchez-Midence, L; Guzmán, T.

Entre la teoría y la práctica de la conservación de los recursos naturales:

las parcialidades I de Totonicapán,

Guatemala. Tecnología en Marcha.Vol.

26, № 3. Pág 50-62

I Con la denominación de parcialidades se conoce en Guatemala a aquellos grupos sociales con vínculos de parentesco pertenecientes a la etnia k'ich'e, que poseen una extensión variable de tierra en común, la cual se caracteriza por presentar áreas con cobertura boscosa con un alto nivel de conservación. Según Palma Murga (Hostnig, 1998), las parcialidades pueden definirse como "una forma de organización social comunitaria en donde el vínculo fundamental de agrupación era el reconocimiento de la existencia de relaciones de parentesco que, con el paso de los años, permitían desarrollar más fuertemente los lazos de identificación grupal, aún cuando los de tipo familiar no continuaran siendo tan directos y lineales". En todo caso, tal y como afirma Carmack (1983) "en las parcialidades los linajes patriarcales tienen preeminencia; los cabeza de linaje tienen bajo su control la tenencia de la tierra de la parcialidad. En la práctica, son grupos sociales cuyo núcleo es un clan patrilineal, es decir, un grupo de parientes con el mismo apellido y afirmando que descienden de un ancestro común".

2 Centro Universitario de Occidente, Universidad de San Carlos de Guatemala. Calle Rodolfo Robles 29-99, Zona I, Quetzaltenango, Guatemala. Correo electrónico: luissanchez@cytcunoc.org

3 Instituto Tecnológico de Costa Rica. Doctorado en Ciencias Naturales para el Desarrollo. Correo electrónico: tjguzman@ gmail.com 


\section{Palabras clave}

Recursos naturales; bosque; conservación ambiental; etnias; identidad; cultura; interculturalidad

\section{Resumen}

La teoría relacionada con la búsqueda de las causas del accionar depredador de las poblaciones humanas sobre los recursos naturales apunta a que aspectos tales como la pobreza, la reducida escolaridad formal, la marginación social, la alta densidad poblacional, el reducido capital humano y el deficiente capital social, así como el nulo acceso al capital, la tierra y el poder político, se constituyen en aspectos que favorecen y potencian tal actitud.

Sin embargo, en Guatemala, grupos humanos agrupados en parcialidades parecieran negar esa teoría, hecho que parece explicarse a través de la conservación de su identidad étnica y de la prevalencia de una cultura particular y diferenciada. Esta realidad debería motivar el surgimiento y fortalecimiento de una corriente intercultural en ese país, que promueva la incorporación de los conocimientos tradicionales de los pueblos originarios en el diseño de nuevas estrategias de conservación ambiental, en la búsqueda de la auténtica raíz de la nacionalidad guatemalteca. Con el presente artículo se busca demostrar que, en algunos grupos sociales, la conservación de los recursos naturales está más relacionada con aspectos culturales que con la presencia o ausencia de elementos tradicionalmente considerados en este tipo de análisis (educación, pobreza, densidad poblacional, capital humano, etc.).

\section{Key words}

Natural resources; forest; environmental conservation; ethnicity; identity; culture; intercultural

\begin{abstract}
The theory regarding the causal search of predatory actions of human populations on natural resources points out that issues such as poverty, formal schooling, social exclusion, population density, human capital and social capital and the no access to land and political power constitute detonating aspects to encourage and strengthen this attitude in any human group. However, groups of people clustered in "parcialidades" seem to disprove this theory, which could be explained by the conservation of their ethnic identity and prevalence of a particular and differentiated culture. This reality should motivate the emergence and strengthening of intercultural power in Guatemala. A kind of power that could made possible to incorporate traditional knowledge of indigenous peoples in the design of new strategies for environmental conservation, and in finding the real root of Guatemalan nationality.

The present article seeks to demonstrate why, in some social groups, conservation of natural resources is more related to cultural aspects than to the presence or absence of items traditionally considered in this type of analysis (education, poverty, density population, human capital, etc.).
\end{abstract}




\section{Introducción}

El deterioro de los recursos naturales, y los efectos sociales, económicos y políticos que conlleva, renueva el planteamiento de una pregunta de inquietante actualidad ¿De qué forma los seres humanos dan valor a sus recursos naturales? Para responder a ella, inicialmente se debe reconocer que los recursos naturales llevan asociados tanto valores intrínsecos como extrínsecos. Los valores intrínsecos se entienden como aquellos que no ofrecen ningún beneficio directo para la humanidad, ${ }^{4}$ al contrario de los valores extrínsecos, que sí le ofrecen beneficios directos (Lennon \& Mathews, 1996). La importancia otorgada a estos valores por parte de cada grupo social particular predispondrá la acción de los individuos frente a los recursos naturales que les rodean.

Sin embargo, el orden de importancia vigente en un momento determinado tampoco es definitivo, puesto que las poblaciones reconfiguran continuamente los patrones de uso de sus recursos naturales, en función de la evolución de elementos contextuales particulares (tanto sociales como ambientales). Es decir, las comunidades modificarán su matriz de oportunidades y restricciones ambientales en virtud de los incentivos o desincentivos que les presionen.

Por otra parte, se debe recordar que las múltiples manifestaciones de crisis diversas (entre ellas la ambiental) demandan la reconstitución de un conocimiento unificado y totalizador de la realidad, la conjunción de las partes de un conocimiento que hasta hoy ha permanecido fraccionado. Desde esa óptica, la interdisciplinariedad se convierte en una necesidad insoslayable, entendiéndola como el proceso a través del cual diferentes disciplinas (tales como la ecología, la geografía, la antropología, la sociología, la economía, la arquitectura, el derecho, etc.), dentro de su especificidad disciplinaria, interactúan en la construcción de una concepción de lo ambiental, generando un proceso de internalización, intercambio y producción de conceptos que promueven una reformulación de los contenidos de estas mismas disciplinas. Así, la interdisciplinariedad teórica se entiende no como el tratamiento común de una temática por diversas disciplinas sino como

4 Por ejemplo, el valor intrínseco del bosque de existir por sí mismo. Estos valores se encuentran asociados con posiciones filosóficas y principios ecológicos profundos (Lennon \& Mathews, 1996). la construcción de un "nuevo objeto científico" a partir de su colaboración (Leff, 2000).

Aunado a esta corriente interdisciplinaria encontramos también la creciente importancia de un enfoque intercultural, el cual pretende lograr la buena convivencia entre las distintas culturas, promoviendo relaciones e intercambios sustentados en oportunidades variadas de comunicación y cooperación (Victorino \& Reyes, 20 I0).

Por otra parte, en el marco de los aspectos que al interior de los grupos humanos estimulan actitudes que propician el deterioro de los recursos naturales, es importante mencionar a algunos autores (entre otros, Guevara, A. y Muñoz, C., 1993; Toledo, C. y Bartra, A. 2000; Fisher, R.J., McGinnis, S., Jackson, W.J., Barrow, E. y Jeanrenaud, S. 2006; Vermulen, S. 2005; Felipe Cárdenas et al. 2000; Lackey, A.S., Burke, R. y Peterson, M., 1987), para quienes existe una relación causa-efecto entre la pobreza y el deterioro ambiental. Es decir, sostienen que a mayor pobreza de las comunidades rurales, mayor deterioro ambiental. Según esta postura, hay tres importantes razones que explican la mayor presión sobre el ambiente de las personas que viven en extrema pobreza: a) el valor relativo del consumo presente respecto al consumo futuro, b) la falta de capital inicial y la escasa información que induce a la toma de decisiones erróneas sobre el uso de los recursos naturales, y c) el empleo de tecnologías que generan impactos ambientales negativos o el abandono de tecnologías conservacionistas.

En todo caso, como afirman estos autores, más que una causa de degradación de los ecosistemas, la pobreza es un mecanismo por medio del cual ciertas condiciones objetivas de supervivencia se transforman en acciones que causan degradación ambiental. Asociado a la pobreza se menciona también un bajo nivel de escolaridad formal, la marginación social, la elevada densidad poblacional, la limitación en el capital humano y el capital social, así como el nulo acceso al capital, la tierra y el poder político, como elementos determinantes en los niveles de deterioro ambiental propiciados por un grupo humano particular.

Un marco analítico interesante para el estudio del nivel de sostenibilidad alcanzado por una comunidad es el propuesto por Mcgregor (Mcgregor, 2003). En su imagen de pirámide (ver Figura I), se transmite la impresión de los dos extremos: una comunidad 


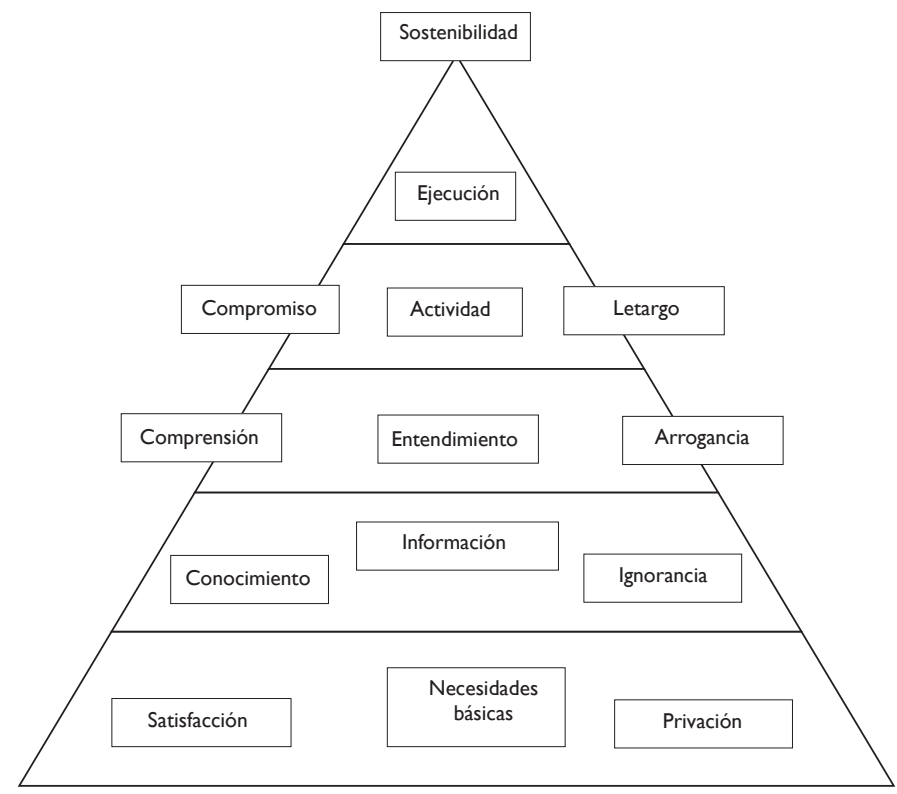

Figura I. Marco analítico para la evaluación y descripción de la sostenibilidad de la comunidad. Fuente: Mcgregor, 2003.

sostenible (en la parte superior de la pirámide) y una comunidad totalmente insostenible (en la parte inferior). Cinco etapas o niveles se requieren para alcanzar la sostenibilidad (de abajo hacia arriba): las necesidades básicas, la información, las actitudes, la actividad y la realización, colocándose en el ápice del modelo la visión de "sostenibilidad" que es compartida por el grupo en cuestión.

Con base en este modelo, una comunidad ubicada en el nivel más alto de la escalera sería aquella que demuestra altas puntuaciones en todas las dimensiones e indicadores. Los niveles presentados son secuenciales, es decir, debe satisfacerse plenamente el nivel de necesidades básicas antes que el nivel de información se vuelva importante, y así sucesivamente.

A través del presente artículo (cuyo contenido proviene de los avances de la investigación doctoral titulada Análisis del sustento socioeconómico, político-organizativo y cultural de la conservación del bosque en las Parcialidades, Totonicapán, Guatemala, en el marco del Doctorado en Ciencias Naturales para el Desarrollo), se pretende verificar la validez de esta información teórica, contrastándola con la realidad particular que se expresa en siete parcialidades estudiadas en el municipio y departamento de Totonicapán, Guatemala: I) parcialidad Ajpacajá, 2) parcialidad Indígena Chuamazán, 3) parcialidad
Menchú Quiacquix, 4) parcialidad Puac, 5) parcialidad Tax, 6) parcialidad Vásquez y 7) parcialidad Velasco. En la Figura 2 se presenta la ubicación de cada una de estas parcialidades en el mapa de la República de Guatemala y en Totonicapán.

Las parcialidades tienen características distintivas comunes, entre las cuales se pueden señalar:

a. Todos sus miembros se reconocen como descendientes de un ancestro común. En otras palabras, todos guardan algún tipo de relación consanguínea, lo cual incrementa la unidad del grupo. Esta unidad étnica también se refleja en la unidad cultural e idiomática de sus miembros.

b. La finalidad de la parcialidad (esto es, la protección del área boscosa comunal) trasciende la búsqueda común de mejoras económicas globales o el incremento de los ingresos monetarios particulares de los socios. Su razón de ser parte de la protección de la herencia de los antepasados, de preservar su relación cultural con la naturaleza y la necesidad de conservar los recursos naturales para beneficio futuro de los miembros de su comunidad.

c. El cuidado y la conservación del área comunal demandan la inversión continua de tiempo y recursos por parte de sus miembros, los cuales son totalmente gratuitos. No existen 


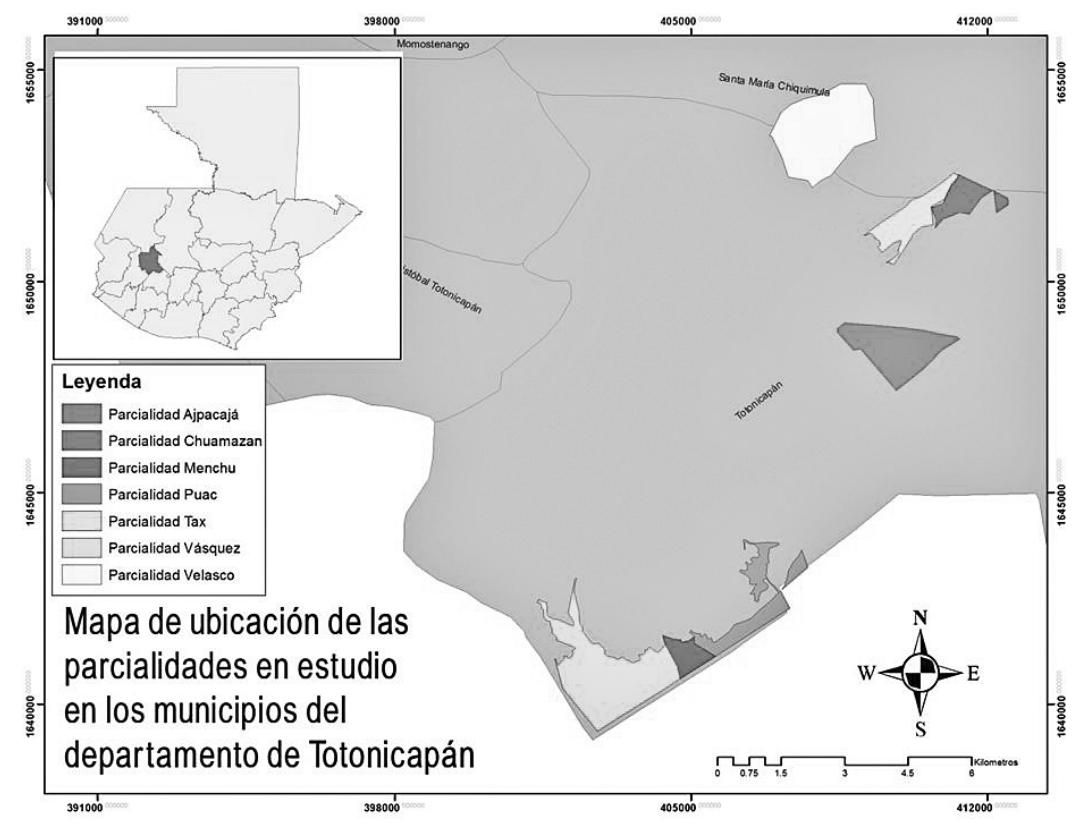

Figura 2. Ubicación de las parcialidades investigadas en el mapa de la República de Guatemala.

Fuente: Elaboración propia. Trabajo de gabinete 2011 .

mecanismos que permitan la recuperación monetaria de esa inversión y, en consecuencia, la participación no está vinculada con la búsqueda del beneficio personal.

d. Las normas y reglamentos organizacionales trascienden el ámbito estrictamente relacionado con las actividades vinculadas a la protección y conservación del bosque, para regular procesos que, en otro ámbito, podrían considerarse estrictamente particulares (como la venta de terrenos de propiedad privada, por ejemplo).

e. El incremento en el número de socios está íntimamente relacionado con la consanguinidad. Esto significa que las posibilidades de acceso para los foráneos es casi imposible.

\section{Materiales y métodos}

La información incluida en este artículo se capturó y procesó durante el período comprendido de julio a noviembre de 201 I, a través de la aplicación de un instrumento de evaluación diseñado para el efecto. Con dicho instrumento se capturó información tanto sobre aspectos sociales (composición de la familia, características de la vivienda, nivel de escolaridad formal, educación informal, migración y sensibilización ambiental) como económicos (ingresos, acceso al crédito y a la tierra) de los miembros, por parcialidad. Para ello se estableció una población muestral por parcialidad (cuyo universo estaba constituido por socios activos que fueran padres de familia), la cual se determinó aplicando la fórmula:

$$
n=\frac{n^{\prime}}{1+n^{\prime} / N}
$$

Quedando el tamaño de dichas muestras establecidas en la forma siguiente: (ver cuadro I).

\section{Resultados}

La información recabada en el área de estudio permite establecer inicialmente los niveles de educación formal alcanzados por los padres de familia, para cada una de las parcialidades estudiadas, la cual se presenta en las figuras 3 y 4.

El contenido de estas figuras permite realizar dos inferencias: la primera está relacionada con el bajo nivel de educación formal que alcanzan los padres de familia, lo cual es causa y efecto de los niveles de pobreza registrados en la zona. La segunda se refiere a que, aún a estos ínfimos niveles de educación formal, puede observarse una diferencia entre hombres y mujeres, lo cual refleja ciertos niveles de 
Cuadro I. Población muestral calculada por parcialidad.

\begin{tabular}{|c|c|c|c|}
\hline Nombre de la parcialidad & $\begin{array}{c}\text { Número total de socios } \\
\text { (condueños) }\end{array}$ & Número total de familias & Tamaño de muestra \\
\hline Parcialidad Ajpacajá & 85 & 84 & 72 \\
\hline Parcialidad Chuamazán & 38 & 38 & 35 \\
\hline Parcialidad Menchú & 240 & 233 & 159 \\
\hline Parcialidad Puac & 647 & 606 & 274 \\
\hline Parcialidad Tax & 90 & 90 & 76 \\
\hline Parcialidad Vásquez & 1367 & 1189 & 352 \\
\hline Parcialidad Velásco & 800 & 738 & 298 \\
\hline * En algunas circunstancias, una misma familia puede incluir a más de un socio (condueño), específicamente en los casos en los \\
que los hijos mayores de edad aún viven con sus padres. En dichos casos, solamente se consideró como (socio) condueño al \\
padre de familia.
\end{tabular}

Fuente: Elaboración propia. Trabajo de gabinete, 201 I.

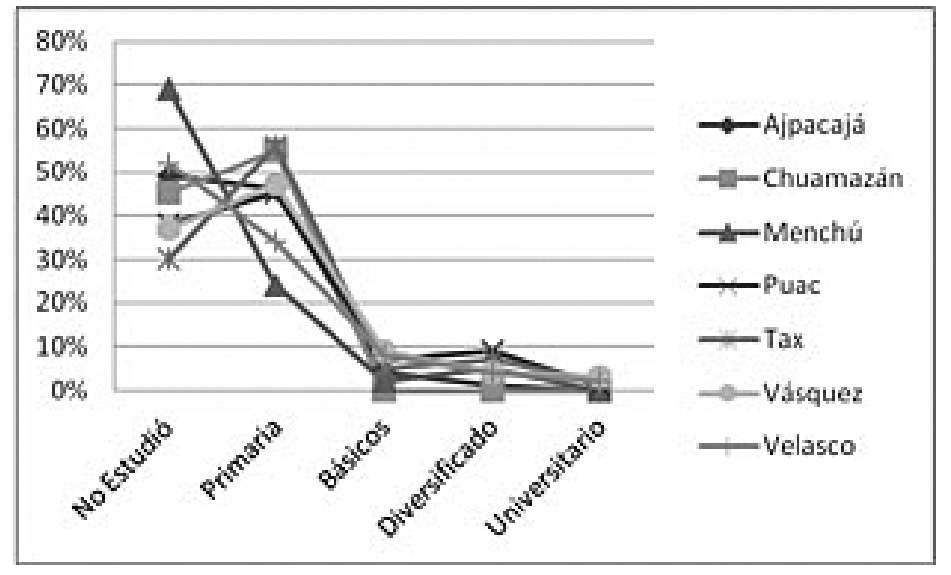

Figura 3. Educación formal alcanzada por los padres de familia (por parcialidad, en porcentajes).

Fuente: Elaboración propia. Trabajo de campo 2011.

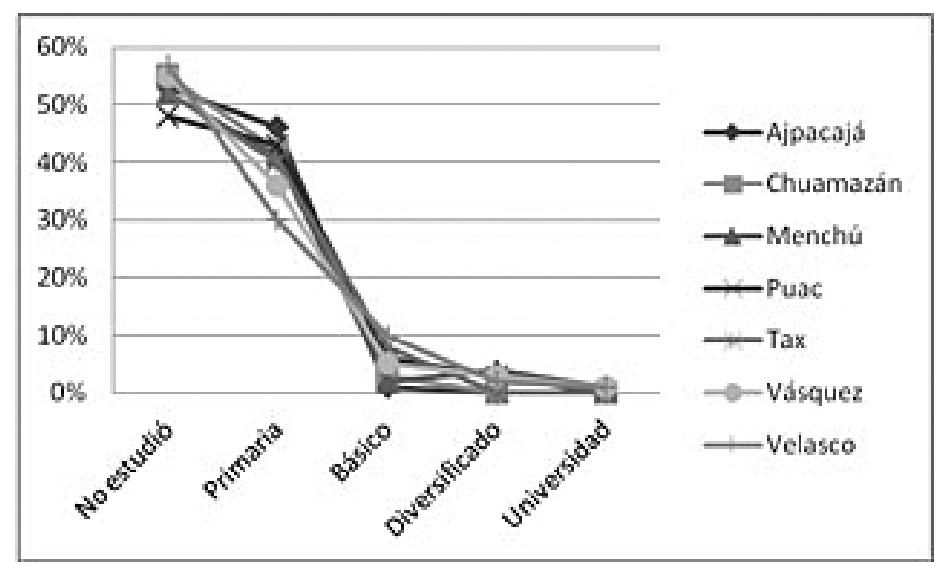

Figura 4. Educación formal alcanzada por las madres de familia (por parcialidad, en porcentajes). Fuente: Elaboración propia.Trabajo de campo 2011 . 
discriminación basada en el género, privilegiando la educación del varón por sobre la de la mujer.

Con relación al análisis de los niveles de educación formal alcanzados por las hijas e hijos de los socios de las distintas parcialidades bajo estudio, se identifica una tendencia clara que orienta a la mejora en los niveles de educación formal inter-generacional, manifestándose un aumento en el nivel promedio de educación que presentan los hijos e hijas con relación al que alcanzaron sus padres, aunque este aún permanece bastante bajo (sexto grado de educación primaria). Además, pese a que parece reducirse en la generación presente, la brecha entre los niveles de educación formal alcanzada por los hombres y las mujeres aún se mantiene. En todo caso, el bajo nivel de educación formal limitaría el acceso al mercado laboral, reduciendo el monto de los ingresos familiares $y$, en consecuencia, y de acuerdo con la teoría presentada al inicio de este artículo, se obligaría a los miembros de estas familias a asumir una actitud depredadora frente a los recursos naturales.

Por otra parte, la carencia de educación formal también puede asociarse con un conocimiento limitado de las funciones benéficas que representa el medio ambiente, las interacciones entre sus elementos y la importancia de su conservación. En este último caso, una actitud de menosprecio e indiferencia hacia el medio ambiente debería prevalecer como una actitud normal, entre los miembros del grupo. En otras palabras, los niveles de educación formal encontrados, en promedio, entre los condueños y sus familias, en las distintas parcialidades estudiadas, no parecen motivar por sí mismos una actitud de protección y conservación de los recursos naturales.

Por otro lado, y de acuerdo con los resultados del $X$ Censo Nacional de Población y $\mathrm{V}$ de Habitación y del XI Censo Nacional de Población y VI de Habitación, realizados en 1994 y 2002, respectivamente, en el municipio de Totonicapán se manifiesta una tasa de crecimiento ínter-censal de 2,46\% anual. De hecho, las familias típicas de los condueños de las distintas parcialidades estudiadas están integradas, en promedio, por seis miembros, el padre, con una edad de 44 años, la madre, con una edad de 42 años, dos hijas en edades de 13 y 18 años, y dos hijos en edades de 14 y 18 años.

En todo caso, una mayor población, de acuerdo con la teoría presentada, implica forzosamente una mayor presión sobre los recursos existentes. Si a esto añadimos los niveles de pobreza presentes en las distintas parcialidades estudiadas, se esperaría la preeminencia de una actitud depredadora hacia el medio ambiente. Así, la alta tasa de crecimiento poblacional presente tampoco parece estimular la conservación del medio ambiente.

Con relación a la pobreza, para el mes de noviembre de 20 I I la canasta básica alimentaria en Guatemala (que incluye el mínimo nutricional que debe ingerir una familia de cinco personas y está compuesta por 26 productos), alcanzó un precio de Q24 I5,00 (US\$309,295), mientras que el monto de la canasta básica vital o ampliada (que comprende más de 400 productos y servicios básicos para una familia de cinco integrantes) alcanzó para el mismo mes un precio de Q4408,03 (US\$564,54).

En promedio, los ingresos familiares mensuales alcanzados por los grupos familiares que integran las distintas parcialidades estudiadas, para ese momento histórico, se presentan en la figura 5.

La información reflejada en la figura 5 señala datos alarmantes sobre los niveles de pobreza que imperan en las distintas parcialidades en estudio. El 94,6\% de los condueños sobrevive con un ingreso mensual que oscila entre Q0,00 y menos de Q2000,00 (de US\$0,00 a menos de US\$256,14), cantidad que no alcanza para cubrir el monto de la canasta básica alimentaria (la cual está estimada para cinco personas, mientras que, en promedio, las familias de los condueños están integradas por seis miembros). Un $5 \%$ de los condueños posee un ingreso mensual que oscila entre Q2000,00 y menos de Q3000,00 (US\$256, I 4 a menos de US\$384,2I ), suficiente para cubrir la canasta básica alimentaria. Finalmente, solo el $0,4 \%$ de los condueños tendría ingresos suficientes para cubrir el precio de la canasta básica vital o ampliada.

La pregunta que queda por responder es la siguiente ¿Cómo puede una familia de seis miembros sobrevivir con menos de Q2000,00 mensuales (US\$256,I4), cubriendo con ello sus necesidades de alimentación, vestido y educación, entre otras? La respuesta a esta pregunta no es sencilla pero se aventuran tres posibilidades, las cuales no resultan excluyentes sino complementarias. La primera se

5 A una tasa de cambio de 7,80822987 quetzales por US $\$$ I, vigente para ese momento histórico. 


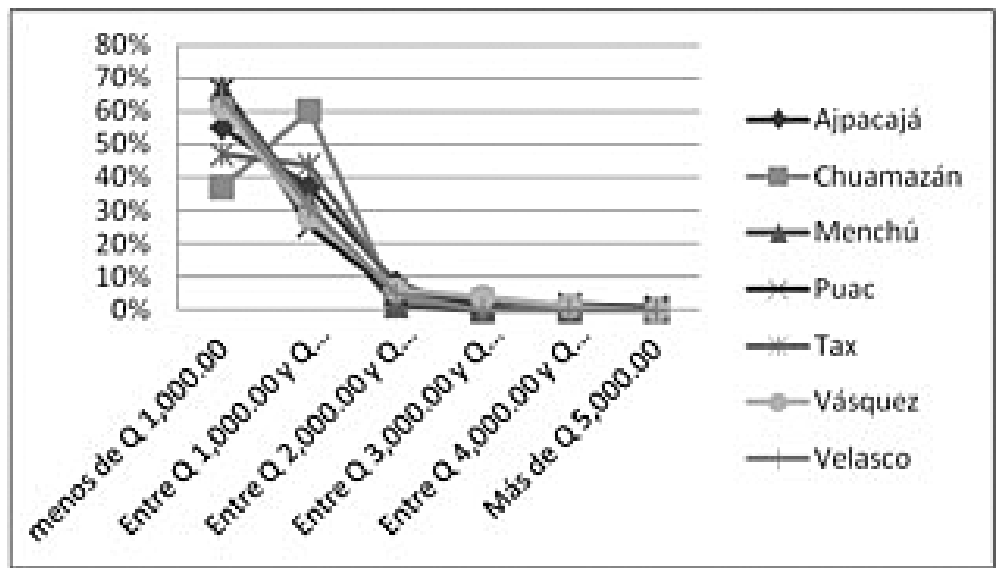

Figura 5. Ingresos económicos familiares mensuales (por parcialidad, en porcentajes).

Fuente: Elaboración propia. Trabajo de campo 2011 .

relaciona con el hecho de que, en condiciones normales, los pequeños productores no tienden a incluir en el monto de sus ingresos los productos no comercializados de la producción de sus parcelas, huertos familiares, aves de corral, árboles frutales, hierbas comestibles o subproductos del bosque (como leña), etc., puesto que se destinan al autoconsumo. Por ello, pese a que estos productos ayudan a complementar los ingresos familiares y poseen un valor monetario, no son contabilizados como tales al momento de hacer un cálculo de los ingresos familiares. La segunda tiene que ver con la solidaridad comunal y el flujo constante de bienes y servicios no monetarios entre los miembros de la comunidad, los cuales contribuyen a superar las deficiencias y limitaciones de diversa índole que se enfrentan y que tienden a agudizarse durante determinadas épocas del año. La tercera está relacionada con la disminución del volumen y calidad de alimentos ingeridos por la familia, lo cual se traduciría en una reducción ostensible de la calidad de vida familiar y en la aparición de problemas tales como la desnutrición infantil. ${ }^{6}$

En cualquier caso, queda claro que la supervivencia de estos grupos familiares solo se posibilita en la medida en que se involucren en la generación de

6 Guatemala presenta una tasa de desnutrición crónica que afecta al $49 \%$ de los niños menores de cinco años. De hecho, es el país latinoamericano con más alta desnutrición crónica y el sexto a nivel mundial. ingresos todos sus miembros, a través de múltiples ocupaciones.

La información presentada sobre los niveles de pobreza que se manifiestan entre los condueños de las distintas parcialidades, de acuerdo con la teoría presentada, no debería permitir que temáticas como la protección ambiental prevalezcan por sobre las necesidades de supervivencia. En ese sentido, una actitud depredadora de los recursos naturales parecería ser la más coherente, puesto que la misma permitiría aumentar, al menos en el plazo inmediato, la posibilidad de acceder a un mayor número de satisfactores para las familias (al mejorar los ingresos a través de la comercialización de elementos de la flora y la fauna presentes en las áreas de propiedad comunal).

Las fuentes del total de ingresos mensuales de que disponen las familias de los condueños de las distintas parcialidades estudiadas provienen, en forma predominante, de la parcela familiar, la venta de jornales, el trabajo formal e informal fuera de la agricultura y la recepción de remesas familiares (principalmente de Estados Unidos de Norteamérica).

Si bien se demuestra la existencia de diversas fuentes de ingreso para los condueños de las distintas parcialidades y los miembros de sus familias, las actividades laborales identificadas presentan, en esencia, tres características comunes: requieren de fuerza de trabajo no calificada, no establecen una 
edad mínima para involucrarse en ellas y otorgan una baja remuneración. Así, tenemos como imagen resultante varios miembros de cada una de las familias de los condueños, de edades diversas, involucrados en actividades que generan ingresos aunque la sumatoria global de estos sigue conformando un monto global escaso, en relación con las necesidades de la mayoría de los grupos familiares estudiados.

Al interior de las parcialidades, las fuentes de ingreso se concentran en la fabricación de artesanías (actividad que absorbe una gran cantidad de fuerza de trabajo, especialmente de las mujeres, los niños y los ancianos), en el trabajo de las parcelas familiares y en la ocasional venta de jornales. Al exterior de las parcialidades, son principalmente el comercio y la venta de jornales las actividades que más fuerza de trabajo demandan.

Con relación al acceso al capital por parte de los condueños de las parcialidades, las fuentes que financiaron los créditos, los montos obtenidos y el destino que se les dio, puede afirmarse que sí han tenido la posibilidad de acceder a capital a través de la obtención de créditos y que el alto porcentaje de condueños que no han disfrutado de él (70\% en promedio) puede atribuirse más a una renuencia personal que a restricciones en el otorgamiento. El hecho de que sea el Sistema Bancario el principal otorgante de los créditos disfrutados (muy por encima de los sistemas tradicionales de crédito local), demuestra que, en su gran mayoría, los condueños poseen los medios que les permiten garantizar al otorgante del crédito la devolución del dinero recibido. ${ }^{7}$

Sin embargo, quizá se encuentre precisamente allí el principal obstáculo para que la mayoría de los condueños se decida a optar por un crédito. Si se estudia el destino predominante de los recursos obtenidos a través del crédito (agricultura, construcción o establecimiento de negocios informales), puede estimarse que el uso de estos no redituará

7 Esta realidad también parece evidenciar la ausencia de medios para demostrar la propiedad legal de los terrenos, predominante en el área rural. El otorgamiento de un crédito dejando en garantía una propiedad demanda que esta última esté registrada. En las áreas rurales, el registro de la tierra no se considera necesario, puesto que todos los miembros de la comunidad conocen al propietario de cada parcela y sus límites. Esta realidad ha limitado la generalización de los créditos (especialmente bancarios) en el área rural. Esta situación, al parecer, fue superada entre los condueños de las parcialidades bajo estudio. en grandes beneficios que aseguren su pago. Por el contrario, este tipo de inversiones conlleva elevados riesgos que, ante la débil capacidad económica de los condueños, puede traducirse fácilmente en incapacidad de pago y, en consecuencia, en la pérdida de la garantía ofrecida. En función de las condiciones en las que se desenvuelven los condueños de las parcialidades estudiadas, la garantía que puede ofrecerse para la obtención de créditos es la parcela familiar. La pérdida de esta debido al impago de un crédito representaría la ruina total para los condueños y sus familias, perdiendo el eje central de su precario equilibrio económico. Esta realidad justifica plenamente la renuencia a adquirir un crédito bancario, salvo situaciones de emergencia inmanejables en sus condiciones normales.

Otro elemento destacable es la participación de organizaciones no gubernamentales (ONG) y cooperativas en el otorgamiento de créditos que, como supuesto, presentan condiciones más accesibles en cuanto al monto de los intereses y los requisitos exigidos. Por otra parte, las familias, como grupo de apoyo en momentos de crisis, también juegan un papel importante en el otorgamiento de créditos. Todas estas posibilidades han hecho que se reduzca seriamente la participación de los prestamistas locales (que hasta hace relativamente poco tiempo eran los principales proveedores de crédito en el área rural y que se caracterizaban por los altos montos de los intereses exigidos).

Los créditos obtenidos han sido empleados en dos rubros fundamentales: la construcción de vivienda y el establecimiento de negocios esencialmente informales. En ambos casos, los volúmenes de inversión han sido relativamente bajos (menores a diez mil quetzales), lo cual se justifica por el tipo de construcción predominante8 y el tipo de negocio que se establece (comercio informal al menudeo). Así, el monto percibido a través de los créditos, y el destino al cual se dedica, no han logrado convertir el acceso al crédito en un medio a través del cual los condueños de las parcialidades mejoren ostensiblemente el monto global de sus ingresos.

8 Con relación a las paredes de las viviendas, el $31 \%$ se construyeron con block, el $62 \%$ con adobe, el $5 \%$ con block y adobe y el $2 \%$ con madera aserrada (tabla). En cuanto al techo, en el $42 \%$ se utilizó teja de barro cocido, en el $42 \%$ lámina metálica, en el $8 \%$ hormigón armado y en el $8 \%$ restante se combinó teja de barro cocido y lámina metálica. 
Lo anterior es importante puesto que, si bien es cierto existe una fuente de capital que podría traducirse en el financiamiento de negocios lucrativos, en la práctica el acceso al crédito no ha establecido a la fecha una diferencia real entre los sujetos que han gozado de él y quienes no lo han hecho. Pese a que podría considerarse que el bajo volumen de los créditos solicitados, así como el destino final asignado a estos, responden esencialmente a la carencia de una visión empresarial por parte de los beneficiarios, la realidad podría ser distinta. Por una parte, el monto del crédito al cual se puede acceder está en relación directa con la capacidad de pago del solicitante y el precio de la garantía ofrecida para tal efecto. En ese sentido, los volúmenes de crédito obtenidos pueden ser el resultado promedio de estas dos condicionantes. Por otro lado, como ya se mencionó, existe una renuencia palpable entre los condueños de las parcialidades a optar por créditos, lo cual podría atribuirse, por una parte, a la necesidad de mantener el control de su limitado equilibrio económico y, por otra, a evitar poner en riesgo sus escasas posesiones. Debemos recordar que la visión de los comunitarios no es estrictamente capitalista $y$, en consecuencia, tienen una racionalidad distinta, enmarcada en una cultura que posee referentes totalmente diferentes a los imperantes en el mundo occidental.

Con relación al acceso a la tierra, debemos señalar que al interior de las comunidades en donde se asientan las parcialidades este es casi inexistente (salvo cuando algún comunitario posee una parcela en propiedad -fuera del área comunal- y está dispuesto a venderla, momento en el cual los comunitarios tienen prioridad de compra). Sin embargo, los comunitarios pueden adquirir parcelas fuera de la parcialidad, lo cual está en función de su disponibilidad económica. Así, se establece que, en promedio, el $46 \%$ del total de condueños de las distintas parcialidades bajo estudio tiene acceso a una parcela de tierra. El uso al cual se destina dicha parcela se registra en la figura 6.

Si bien es cierto existe un elevado número de condueños que poseen una parcela familiar fuera del área de la parcialidad (el $97 \%$ de la cual es poseída en propiedad) y que un $87 \%$ de estas parcelas son destinadas a trabajos agrícolas, su producción solamente aporta en promedio el $23 \%$ de los ingresos mensuales familiares de los condueños. Esto puede atribuirse, por una parte, al reducido tamaño promedio de las parcelas $\left(1,079.604 \mathrm{~m}^{2}\right.$ cada una de ellas) $y$, por otra, a los rendimientos obtenidos en el cultivo predominante (7.35 quintales de maíz por cada una de estas parcelas). Esta realidad justifica la búsqueda de ingresos complementarios en otras actividades económicas. En cualquier caso, el tamaño de las parcelas poseídas y su uso actual no contribuyen en forma determinante a solucionar la difícil situación económica por la que atraviesan la mayoría de condueños de las parcialidades estudiadas.

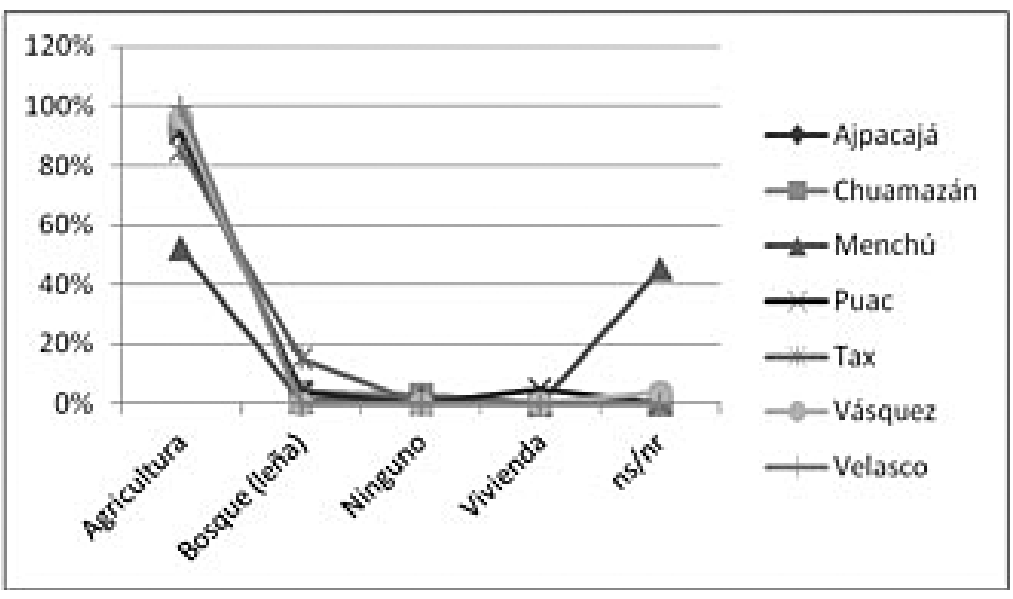

Figura 6. Uso asignado a las parcelas ubicadas fuera de las parcialidades (por parcialidad, en porcentajes). 
Los resultados de todos estos aspectos socioeconómicos en las parcialidades parecerían configurar grupos humanos que, bajo la presión constante de las necesidades de supervivencia, depredan sus recursos naturales, empleándolos como un medio para cubrir sus carencias. En otras palabras, no debería existir ninguna diferencia entre estas parcialidades y el promedio nacional guatemalteco con relación a la conservación de los recursos naturales. Es más, aspectos como el crecimiento de la población y la pobreza (que se agudizan más en este lugar que en otras partes del país) deberían ejercer una fuerte presión para depredar los recursos naturales, más que para conservarlos.

¿Qué es lo que motiva a los individuos miembros de las parcialidades a conservar sus recursos naturales bajo condiciones económicas tan precarias? Inicialmente, debemos considerar su comportamiento como grupo y no como individuos. Es decir, se entiende que la pertenencia de los individuos a este grupo social (la parcialidad) no solamente les impide tomar decisiones en forma aislada sino que las decisiones que cada uno de ellos adopta están siempre configuradas por las decisiones que toman los demás miembros del grupo y por el grupo como un todo (McCay \& Jentoft, 1997). Además, para este caso particular, y puesto que los miembros de las parcialidades comparten también la misma comunidad, la misma historia, la misma lengua y el mismo grupo étnico, prevalecen entre ellos también las mismas normas morales y sociales que, en gran medida, influyen y guían su comportamiento.

En este orden de ideas, las instituciones que un grupo cualquiera crea para manejar o depredar los recursos naturales de los que dispone están enraizadas en el sistema social y cultural de la comunidad. Así, las decisiones que toman los miembros de una comunidad particular con respecto al uso de los recursos naturales responden no solo al incentivo de maximizar las ventajas individuales sino también a factores sociales, culturales y políticos que trascienden al individuo.

Así pues, para este caso particular, son los aspectos culturales los que, al situarse por encima de las limitaciones económicas, orientan la conservación de los recursos naturales. Sin embargo, la pertenencia a estos grupos sociales debe proveer a sus miembros otros beneficios prácticos, más allá de la simple satisfacción de proteger los recursos heredados por los antepasados por motivos culturales. En efecto, las limitantes económicas, políticas y sociales que experimentan los condueños de las parcialidades atentan directamente contra sus posibilidades de supervivencia individual, familiar y comunitaria. Por ello, y ante la ausencia de posibilidades que les permitan mejorar ostensiblemente sus ingresos en el corto plazo, la solidaridad comunal resulta fundamental. El disfrute de dicha solidaridad está íntimamente ligado al reconocimiento social, es decir, a la valoración que los miembros de la comunidad realizan sobre cada uno de sus integrantes, en función del servicio que ha prestado en pro del beneficio comunal. ${ }^{9} Y$ es precisamente ese elevado nivel de solidaridad lo que posibilita la supervivencia individual y colectiva, convirtiendo la parcialidad en causa y producto de ella.

Sin embargo, aún queda por responder una pregunta ¿Cuál es la diferencia entre este grupo humano particular (las parcialidades) y los grupos estudiados por los teóricos que establecieron una relación entre el deterioro de los recursos naturales y una serie de factores económicos y sociales? Las diferencias que pueden identificarse tienen una doble vertiente: por una parte, el hecho de que los miembros de las parcialidades no se encuentren totalmente sometidos al mercado capitalista y que, en consecuencia, el individualismo, el consumismo, la búsqueda del enriquecimiento y la acumulación, y la certeza de que la utilidad de la naturaleza se mide únicamente en función de los bienes económicos que se pueden extraer de ella, no gobiernen aún su comportamiento social, lo cual posibilita la toma de decisiones "irracionales" desde la perspectiva capitalista, empleando otro tipo de referentes. Por otro lado, la prevalencia de una cultura que valora los recursos naturales, no solamente porque son herencia de los antepasados sino porque su conservación está íntimamente ligada a la supervivencia comunal (más que en el ámbito físico, en el ámbito de la identidad, del "ser", de conservar lo que son y lo que los diferencia de otros grupos étnicos y sociales). Lo anterior permite afirmar que para grupos humanos inmersos en el sistema capitalista, las afirmaciones emanadas de los teóricos que hemos analizado pueden resultar

9 Esto parece ratificarse con la participación activa que los miembros de las parcialidades realizan gratuitamente, ya sea en los cargos de la Junta Directiva o en las actividades cotidianas de conservación y protección del bosque, trabajos que no son remunerados. 
totalmente válidas, independientemente de su lugar de ubicación (lo que podría denominarse una "verdad universal"). Sin embargo, estos criterios carecen de validez cuando lo que predomina en los grupos humanos son percepciones distintivas, sustentadas en culturas no depredadoras sino armoniosas con los recursos naturales.

Desde esta perspectiva, la penetración paulatina del capitalismo y de la cultura occidental (a través del retorno de los migrantes, el mayor contacto con el mercado o la influencia de la educación formal o los medios de comunicación masiva -internet, televisión, radio- en las nuevas generaciones, que idealizan la cultura occidental mostrándola como la única forma "civilizada"), combinada con la indiferencia del Estado, generan inquietud sobre el futuro de estas organizaciones, la cual está íntimamente ligada a la preservación de su identidad étnica. La única posibilidad real de conservación de estas organizaciones étnicas atraviesa necesariamente por un cambio en la percepción de la realidad étnica en el país, evolucionando desde un enfoque multicultural a uno intercultural.

En efecto, el creciente deterioro ambiental que experimentan países como Guatemala demanda reflexionar sobre la importancia de incorporar el conocimiento tradicional de los pueblos indígenas como parte sustancial (por primera vez) del desarrollo nacional, buscando medios innovadores que faciliten la convalidación del carácter científicopráctico de los saberes populares que se derivan de las tradiciones de los pueblos indígenas (Victorino, en Victorino y Reyes, 20 I0).

Debe destacarse el hecho de que los procesos reales de reapropiación de la naturaleza y de la gestión ambiental participativa deben implicar, más allá de la articulación interdisciplinaria de conocimientos, una hibridación entre ciencias, tecnologías, prácticas tradicionales y saberes populares, así como un diálogo intercultural en un campo antagónico marcado por sentidos diversos e intereses diferenciados (Leff, en Leff, 2000).

Esta nueva corriente que promueve la necesidad de un diálogo multicultural que propicie una verdadera interculturalidad ${ }^{10}$ choca de frente con

I0 La interculturalidad no es sinónimo de indigenismo, puesto que el término se refiere básicamente a la relación entre culturas y esto incluye a la población no indígena. la desigualdad y sometimiento de los pueblos indígenas en Guatemala, vigentes desde la época colonial y que han perdurado hasta el presente (aunque han evolucionado las prácticas excluyentes). Esta nueva visión plantea la ruptura de la práctica histórica vigente, orientada a desarraigar la cultura originaria (en uso desde hace más de 500 años), considerando en su lugar necesario y urgente aprender a reconocer el origen de nuestra cultura para aceptar las diferencias entre grupos, que se traducirá en el respeto a sus derechos." Se concibe así una nueva orientación que se propone modificar las formas de abordar y atender la diversidad cultural (Victorino, en Victorino y Reyes, 20 I0).

De acuerdo con Essomba (2006), la interculturalidad no debe ser solamente un discurso sino también una práctica; no es responsabilidad de los políticos ni de la administración pública sino de la sociedad civil; fundamenta su acción en el reconocimiento de un axioma básico: todos los seres humanos somos iguales en esencia y diferentes en existencia. Así, las poblaciones indígenas y no indígenas deben ser educadas desde la perspectiva de aceptación de las diferencias. Al mismo tiempo, debe estimularse el conocimiento, la valoración y la recuperación de los conocimientos ancestrales que forman parte de nuestra riqueza cultural.

Únicamente una adecuada convivencia entre las distintas culturas que integran hoy la nación guatemalteca, que sustente las relaciones e intercambios en un marco de respeto, cooperación y comunicación, posibilitará el enriquecimiento de la actitud y estrategia nacionales relacionadas con la conservación de los recursos naturales (a través de la incorporación de los conocimientos tradicionales propiedad de los grupos indígenas guatemaltecos), facilitando el acceso a un auténtico reconocimiento de nuestras raíces y, en consecuencia, a la posibilidad de construir un futuro prometedor para todos sus ciudadanos.

II Existe detrás de todo esto la comprensión de que cuando los conocimientos "universales" se transmiten a otros grupos étnicos como verdades únicas y sus conocimientos locales se tratan como alegorías innecesarias al "desarrollo necesario", es posible que se esté negando sistemáticamente a estos colectivos la posibilidad de construir un futuro digno y sostenible, pues es muy probable que gran parte de los conocimientos necesarios para la continuidad de la vida se encuentren entre los saberes ya manejados en las comunidades (Leal y Bonilla, 2005). 


\section{Conclusiones}

La información contenida en este artículo permite verificar que en algunos grupos humanos (como son las parcialidades en el caso particular analizado) en los cuales predominan altos niveles de pobreza, baja escolaridad formal, alta densidad poblacional y elevada presión sobre la tierra, pueden aún encontrarse vigentes marcos culturales que propician una convivencia armónica con el medio ambiente (bosques, en este caso), expresados en el logro de altos niveles de conservación. Esta realidad debería estimular, y no solamente para el caso guatemalteco sino para otras realidades similares, la ocurrencia de un auténtico diálogo intercultural, que permita considerar estos marcos culturales en el diseño de las políticas nacionales que buscan la preservación de los recursos naturales.

Actualmente, existe un creciente involucramiento a nivel mundial de los distintos grupos étnicos y comunidades indígenas en numerosos programas y proyectos orientados a la conservación ambiental de sus territorios, además de la emergencia de un movimiento social que abandera la defensa de los recursos naturales que precisamente se apoya en los derechos culturales de las poblaciones, promoviendo el establecimiento de un dialogo de saberes -de interpretaciones e intereses sobre el ambiente- que sustenta los procesos de reapropiación de la naturaleza y de la cultura. Todo ello posibilita el hecho de que las poblaciones autóctonas dejen de ser un mero objeto de estudio de la antropología, para convertirse en actores vivos de una política de la sustentabilidad (Leff, en Leff, 2000).

\section{Bibliografía}

Carmack, R.M. (1983). El Título de Totonicapán. México: CEMUNAM.

Essomba, M.A. (2006). Liderear Escuelas Interculturales e Inclusivas. Equipos directivos y profesorado ante la diversidad cultural y la inmigación. Barcelona: Graó.

Felipe Cárdenas, T. (ed., comp.) (2000). La conservación y la producción por parte de las comunidades locales en la cuenca media del río Chicamoya (Boyacá), Colombia. Santa
Fe de Bogotá: Instituto de Estudios Ambientales para el Desarrollo (IDEADE)-Facultad de Estudios Ambientales y Rurales, Pontificia Universidad Javeriana.

Fisher, R.J., McGinnis, S., Jackson, W.J., Barrow, E. \& Jeanrenaud, S. (2006). Pobreza y Conservación. Paisajes, Pueblos y Poder. Gland: IUCN.

Guevara, A., Muñoz, C. (1993). La dinámica de la pobreza y el medio ambiente. México: Centro de Estudios Económicos, Colegio de México y Unidad de Análisis Económico de la Secretaría de Desarrollo Social.

Hosting, R. (comp.) (1998). Esta tierra es nuestra. Compendio de fuentes históricas sobre denuncias, medidas y remedidas, composiciones, titulaciones, usurpaciones, desmembraciones, litigios, transacciones y remates de tierra (años 1567 1993). Tomo III. Municipio de San Miguel Totonicapán y sus parcialidades. Guatemala. Quetzaltenango: Cooperación para el Desarrollo Rural de Occidente, Totonicapán e Instituto para la Cooperación Internacional.

Lackey, A.S, Burke, R. \& Peterson, M. (1987). Healthy communities: the goal of community development. Journal of the Community Development Society 18. 1-17.

Leal, D. \& Bonilla, O. (2005). Aruanda. Elementos Preliminares para la Creación de la paz. Quito: Centro de Estudios para la Paz.

Leff, E. (coord.) (2000). Los problemas del conocimiento y la perspectiva ambiental del desarrollo. 2 ed. México: Siglo XXI Editores.

Lennon, J. \& Mathews, S. ( 1996). Cultural Landscape Management: guidelines for identifying, assessing and managing cultural landscapes in the Australian Alp National Parks. Australian Heritage Working Group.

McCay, B.J. \& Jentoft, S. (1997). Market or community failure? Critical perspectives on common property research. Human Organization, Vol. 57, No I. 55-I 17.

Mcgregor, C. (2003). Working towards sustainability in small towns: a perspective from northern Australia. International Journal of Environment and Sustainable Development, Vol. 2, No 4, p. 35-78.

Toledo, C. \& Bartra, A. (eds.) (2000). Del círculo vicioso al círculo virtuoso. Cinco miradas al desarrollo sustentable de las regiones marginadas. México: Plaza y Valdez.

Vermulen, S. (2005). Power Tools: Handbook to Tools and resources for policy in natural resources management. International Institute for Environment \& Development. United Kingdom: Cambridge University Press.

Victorino, L. \& Reyes, A. (coord.) (20/0). Epistemología, Educación Agrícola e Interculturalidad: Una tríada posible para coadyuvar al desarrollo sustentable. México: Universidad Autónoma de Chapingo. 